\title{
Nerve Conduction Studies of Peripheral Motor and Sensory Nerves in the Subjects With Prediabetes
}

\author{
Nikhil Rathi ${ }^{a}$, Bharati Taksande ${ }^{\mathrm{b}, \mathrm{c}}$, Sunil Kumar ${ }^{\mathrm{a}}$
}

\begin{abstract}
Background: Prediabetes is a condition in which the fasting blood glucose is elevated above normal levels but not high enough to be classified as diabetes mellitus. There is clear association between sensory polyneuropathy and diabetes mellitus. But association of neuropathy in prediabetes is still not clear. Forty years ago, Ellenberg suggested that neuropathy may indeed occur in prediabetes.
\end{abstract}

Methods: In the present study, we attempted to study the changes in nerve conduction studies in the patients with glucose intolerance, i.e. impaired fasting blood glucose or impaired glucose tolerance in a tertiary care rural hospital. The study included 65 cases (prediabetic) and 65 controls. Nerve conduction studies were performed on right tibial nerve and right sural in lower limb, with surface recording, using the standardized technique.

Results: The compound muscle action potential (CMAP) and nerve conduction velocity $(\mathrm{NCV})$ of right tibial nerve were significantly reduced in the cases as compared to controls and were found to be statistically significant suggesting motor axonal neuropathy. The sensory nerve action potential (SNAP) and NCV of right sural nerve were significantly reduced in the cases as compared to controls which were found out to be statistically significant suggesting that cases had sensory axonal neuropathy.

Conclusion: All the patients of prediabetes should also be screened for neuropathy. We conclude prediabetic stage is risk factor for development of neuropathy, with hyperglycemia being acting via same mechanism as in cases of diabetes. Prediabetes patients are at risk of developing full blown neuropathy. Early metabolic aberrations as seen in impaired glucose tolerance may lead to changes in the nerve conduction.

Keywords: Neuropathy; Prediabetes; Nerve conduction studies

Manuscript submitted August 21, 2019, accepted September 4, 2019

aDepartment of Medicine, JNMC, Sawangi (Meghe), Wardha, Maharashtra, India

bDepartment of Medicine, MGIMS, Sewagram, Wardha, Maharashtra, India ${ }^{\mathrm{c} C}$ Corresponding Author: Bharati Taksande, Department of Medicine, MGIMS, Sewagram, Wardha, Maharashtra, India. Email: bharati.taksande@gmail.com

doi: https://doi.org/10.14740/jem602

\section{Introduction}

Prediabetes is a condition in which the fasting blood glucose is elevated above normal levels but not high enough to be classified as diabetes mellitus. There is clear association between neuropathy and overt diabetes mellitus. But association of neuropathy in prediabetes is still not clear. There is an increasing evidence that patients with milder degrees of abnormal blood glucose, including impaired glucose tolerance (IGT) and impaired fasting glucose (IFG) also called as prediabetes, are also at risk of developing full blown polyneuropathy $[1,2]$.

Forty years ago, Ellenberg suggested that neuropathy may indeed occur in prediabetes; however, factors other than hyperglycemia may cause the neuropathy [3]. The recent resurgence of interest of IGT in neuropathy is based on four separate studies of patients with small-fiber neuropathy of idiopathic origin, where the prevalence of IGT was found to be $34-35.6 \%$, three times more than the prevalence in age-matched control subjects. Two population-based studies have assessed the prevalence of neuropathy in IGT: the San Luis Valley (USA) [2] and the MONICA/KORA Augsburg (Germany) studies [4]. Remarkable similar results of these two epidemiologic surveys were found with neuropathy present in $26-28 \%$ of diabetic subjects, $11-13 \%$ of IGT and $4-8 \%$ of the non-diabetic populations.

Early metabolic aberrations as seen in IGT may lead to changes in the nerve conduction. Results from the studies in the Caucasian population have shown that IGT is associated with peripheral nerves dysfunction $[5,6]$. However, there are not many studies in India, which have looked into the nerve conduction changes in IGT. In the present study, we attempted to study the changes in nerve conduction velocity (NCV) and compound muscle action potential (CMAP) and sensory nerve action potential (SNAP) in the patients with glucose intolerance, i.e. impaired fasting blood glucose or impaired glucose tolerance in a tertiary care rural hospital.

The aim of the study was to look for changes in nerve conduction studies in the subjects of prediabetes and control group. The objectives were: 1 ) to study motor nerve conduction changes in prediabetes; 2) to study sensory nerve conduction changes in prediabetes; 3 ) to study risk factors associated with neuropathy.

\section{Materials and Methods}

\section{Study design and setting}

A prospective case-control study was carried out for a period 
Table 1. Baseline Characteristics of Cases and Controls

\begin{tabular}{lll}
\hline Parameter & Control group $(\mathbf{n}=\mathbf{6 5})$ & Case group $(\mathbf{n}=\mathbf{6 5})$ \\
\hline Age, years & 48.80 & 43.06 \\
Males, $\mathrm{n}$ & 38 & 38 \\
Females, $\mathrm{n}$ & 27 & 27 \\
Systolic blood pressure, mm Hg & $126.18 \pm 7.69$ & $124.95 \pm 7.73$ \\
Diastolic blood pressure, mm Hg & $80.44 \pm 4.52$ & $80.18 \pm 4.26$ \\
Body mass index, kg/m ${ }^{2}$ & $24.15 \pm 2.57$ & $24.39 \pm 3.26$ \\
Total cholesterol, mg/dL & $171.66 \pm 23.03$ & $173.52 \pm 37.05$ \\
High-density lipoprotein, mg/dL & $40.03 \pm 6.11$ & $39.15 \pm 7.41$ \\
Triglycerides, mg/dL & $120.90 \pm 37.78$ & $137.06 \pm 32.22$ \\
Low-density lipoprotein, mg/dL & $106.26 \pm 33.97$ & $126.03 \pm 32.07$ \\
Fasting blood sugar, mg/dL & $87.29 \pm 6.00$ & $111.18 \pm 6.02$ \\
Oral glucose tolerance test (2-h sample), mg/dL & $120.93 \pm 10.30$ & $155.61 \pm 15.05$ \\
\hline
\end{tabular}

of 2 years in the Department of Medicine at rural teaching and tertiary care hospital after getting permission from the Institutional Ethics Committee. The study included 65 cases (prediabetic subjects) and 65 controls who were admitted to the hospital. All procedures performed in studies involving human participants were in accordance with the ethical standards of the institutional and/or national research committee and with the 1964 Helsinki declaration and its later amendments or comparable ethical standards.

\section{Inclusion and exclusion criteria}

According to American Diabetic Association, all the consecutive patients of prediabetes, i.e. IFG 100 to $125 \mathrm{mg} / \mathrm{dL}$ and/ or IGT 140 to $199 \mathrm{mg} / \mathrm{dL}$, and age-matched controls, were included.

Exclusion criteria were: 1) patients who denied consent to be a part of study; 2) alcoholic patients; 3) patients with chronic kidney disease and thyroid disease; 4) $\mathrm{K} / \mathrm{C} / \mathrm{O}$ diabetes mellitus (on insulin therapy or non-insulin therapy or life style management); 5) patients with macrocytic hypochromic anemia; 6) patients on phenytoin, anti-retroviral therapy, anti-tubercular treatment; 7) patients having malignancy; 8) patients who were critically ill that they cannot be transferred for performance of nerve conduction study; 9) patients with trauma to the lower limbs.

All the subjects after giving consent were subjected for nerve conduction studies.

\section{Methods}

The nerve conduction studies were done by trained technician on RMS-NCV-EMG machine and the results were interpreted by a trained physician. Both the technician and the physician were blinded to the cases and controls. Nerve conduction studies were performed on right tibial nerve for motor neu- ropathy and right sural for sensory neuropathy in lower limb, with surface recording, using the standardized technique. The following parameters on nerve conduction study were evaluated: 1) CMAP, peak to peak amplitude; 2) SNAP, peak to base amplitude; 3) NCV. Nerve conduction of sural nerve was recorded by the surface electrode between lateral malleolus and tendoachilles. The nerve was stimulated antidromically 10 - 16 $\mathrm{cm}$ proximal to the recording electrode, distal to lower border of gastrocnemius at the junction of middle and lower third of leg. Normal NCV for sural nerve is greater than $40 \mathrm{~m} / \mathrm{s}$ and amplitude of SNAP is more than $7 \mu \mathrm{V}$.

For tibial nerve conduction, the active surface electrode was placed on the abductor hallucis slightly below and anterior to navicular tuberosity. Surface stimulation was used behind and proximal to the medial malleolus and the popliteal fossa along the flexor crease of the knee slightly lateral to the midline of the popliteal fossa. Normal NCV for tibial nerve is greater than $50 \mathrm{~m} / \mathrm{s}$ and amplitude of CMAP is more than $8 \mu \mathrm{V}$.

Based on the results of the above parameters, the trained physician made a diagnosis of neuropathy. Accordingly, sensory neuropathy was defined if any one or more than one of the findings will be present in sural nerve, i.e. reduced SNAP $(<$ $7 \mu \mathrm{V})$ or reduced conduction velocity $(<40 \mathrm{~m} / \mathrm{s})$. Motor neuropathy was defined if any one or more than one of the findings will be present in tibial nerve, i.e. reduced CMAP $(<8 \mu \mathrm{V})$ and reduced conduction velocity $(<50 \mathrm{~m} / \mathrm{s})$.

\section{Results}

The baseline characteristics of the prediabetes subjects and the controls are shown in Table 1. The results of the nerve conduction studies are shown in Table 2.

The mean CMAP in control was found out to be $12.34 \pm$ $5.28 \mu \mathrm{V}$, while that in cases was $8.50 \pm 3.04 \mu \mathrm{V}(\mathrm{P}=0.0009)$. The mean NCV in controls was found out to be $62.76 \pm 12.82$ $\mathrm{m} / \mathrm{s}$, while that in cases was $51.34 \pm 13.29 \mathrm{~m} / \mathrm{s}(\mathrm{P}=0.0009)$. 
Table 2. Comparison of CMAP, SNAP and NCV of Tibial and Sural Nerves in Cases and Controls

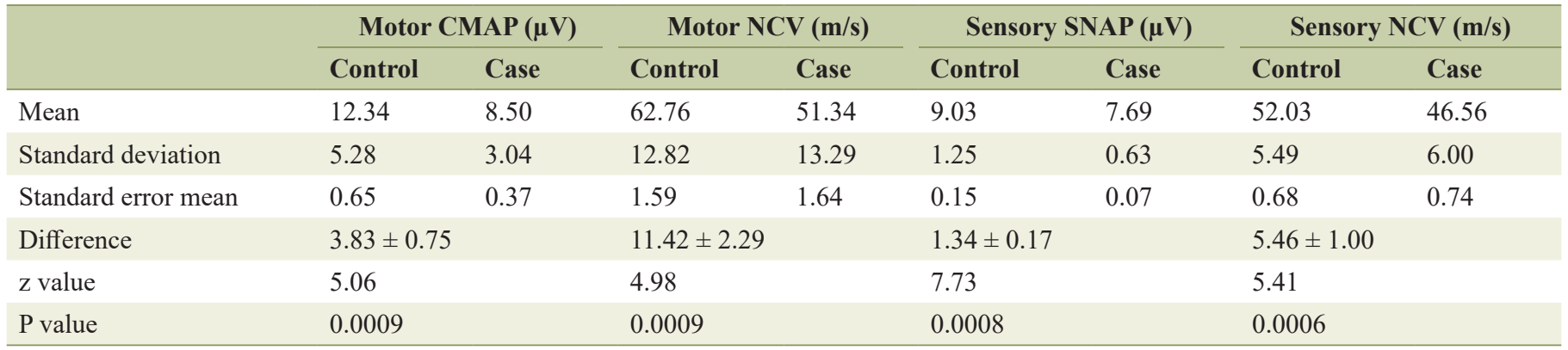

The CMAP and NCV of right tibial nerve were significantly reduced in the cases as compared to controls which were found out to be statistically significant, suggesting that cases had motor axonal neuropathy. CMAP: compound muscle action potential; NCV: nerve conduction velocity; SNAP: sensory nerve action potential.

The SNAP and NCV of right sural nerve were significantly reduced in the cases as compared to controls which were found out to be statistically significant suggesting that cases had sensory axonal neuropathy. The mean SNAP in controls was found out to be $9.03 \pm 1.25 \mu \mathrm{V}$, while that in cases was $7.69 \pm 0.63 \mu \mathrm{V}(\mathrm{P}=0.0008)$. The mean $\mathrm{NCV}$ in controls was found out to be $52.03 \pm 5.49 \mathrm{~m} / \mathrm{s}$, while that in cases was 46.56 $\pm 6.00 \mathrm{~m} / \mathrm{s}(\mathrm{P}=0.0006)$.

\section{Discussion}

In our study, we found there was significant slowing down of both sensory and motor nerve conduction velocities in subject group of prediabetes as compared to the control group. Also there were reductions in CMAP and SNAP in prediabetic group as compared to control healthy group. The nerve conduction study values were not decreased enough to the level for the diagnosis of peripheral neuropathy. However, values were diminished as compared to controls signifying that the pathologic process of neuropathy has started.

Ziegler et al [4] in their study found out that IGT was associated with more incidence of both motor and sensory neuropathy as compared to healthy adults. Barr et al [7] also in their study found out that IGT and IFG were associated with neuropathy. Im et al [8] similarly in their study also confirmed that the SNAP amplitude was significantly smaller in cases of IGT as compared to healthy group. Also tibial nerve CMAP amplitude was smaller in case of IGT as compared to healthy group significantly.

Singleton and Smith $[3,9]$ reviewed many studies regarding neuropathy in prediabetes and came to the conclusion that most patients with IGT and associated neuropathy have a symmetric, distal sensory polyneuropathy with prominent neuropathic pain.

After extensive search, we could find only one Indian study documenting the nerve conduction studies in prediabetes. Viswanathan et al [10] in their study found out that there was significant slowing of motor conduction velocity in IGT subjects as compared to the control healthy subjects. However, they did not find any significant slowing in the sensory nerve conduction velocity. Also there was no significant decrement in the motor and sensory amplitudes in IGT cases.

IGT neuropathy is phenotypically similar to early diabetic neuropathy, which also causes sensory symptoms, including pain, and autonomic dysfunction. The key factor for its development is postprandial hyperglycemia, which is neurotoxic via a number of mutually additive mechanisms: increased oxidative stress, endothelial dysfunction, activation of protein kinase $\mathrm{C}$ and of the polyol pathway, culminating in impairment of mitochondrial function and neuronal metabolism with direct osmotic injury and DNA damage. Other pathogenic factors include dyslipidemia and the metabolic syndrome. The cornerstone of management is improved glycemic control.

In our study, we found out that there was increased lowdensity lipoprotein (LDL) and triglycerides (TG) in prediabetic group as compared to healthy control group which was statistically significant. This was similar to the findings in various other studies done previously.

Lupachyk et al [11] in their study evaluated the roles of IGT versus dyslipidemia in prediabetic neuropathy using Zucker fatty $(\mathrm{fa} / \mathrm{fa})$ rats. They found out that the niacin derivative acipimox, which reduced serum insulin, non-esterified fatty acid (NEFA) and TG concentrations without affecting IGT and total and very low-density lipoprotein (VLDL), LDL cholesterol concentrations, corrected peripheral nerve oxidative nitrosative stress, sensory nerve conduction velocity (SNCV) deficit, and alleviated changes in behavioral measures of sensory function associated with a metabolic condition mimicking prediabetes and obesity in humans. This was the first of the kind study which provided the first experimental evidence for a key role of hypertriglyceridemia and/or increased fatty acid concentrations in peripheral nerve dysfunction associated with prediabetes.

Therefore, deranged lipid profile can act as an independent factor leading to neuropathy besides hyperglycemia.

Prediabetes is the stage when the patient has not been overtly diabetic. It is a controversy whether neuropathy develops in cases of prediabetes. Various studies point out that prediabetic stage is risk factor for development of neuropathy, with hyperglycemia being acting via same mechanism as in cases of diabetes. Our study also concluded that prediabetic subjects are also at risk for developing neuropathy. 


\section{Acknowledgments}

We extend our thanks to all the subjects (cases and controls), phlebotomist and the lab technician to help us to complete this study.

\section{Financial Disclosure}

There is no funding agency who has supported us to carry out this study.

\section{Conflict of Interest}

There is no conflict of interest.

\section{Informed Consent}

Informed consent was obtained from all individual participants included in the study.

\section{Author Contributions}

Dr Nikhil collected the sample; Dr Bharati wrote the manuscript; Dr Sunil Kumar did the statistics.

\section{References}

1. Report of the expert committee on the diagnosis and classification of diabetes mellitus. Diabetes Care. 1997;20(7):1183-1197.

2. Franklin GM, Kahn LB, Baxter J, Marshall JA, Hamman RF. Sensory neuropathy in non-insulin-dependent diabetes mellitus. The San Luis Valley Diabetes Study. Am J Epidemiol. 1990;131(4):633-643.

3. Singleton JR, Smith AG. Neuropathy associated with prediabetes: what is new in 2007? Curr Diab Rep. 2007;7(6):420-424.

4. Ziegler D, Rathmann W, Dickhaus T, Meisinger C, Mielck A, the KORA Study Group. Prevalence of polyneuropathy in pre-diabetes and diabetes is associated with abdominal obesity and macroangiopathy: the MONICA/ KORA Augsburg Surveys S2 and S3. Diabetes Care. 2008;31(3):464-469.

5. Singleton JR, Smith AG, Bromberg MB. Painful sensory polyneuropathy associated with impaired glucose tolerance. Muscle Nerve. 2001;24(9):1225-1228.

6. Sumner CJ, Sheth S, Griffin JW, Cornblath DR, Polydefkis $\mathrm{M}$. The spectrum of neuropathy in diabetes and impaired glucose tolerance. Neurology. 2003;60(1):108-111.

7. Barr EL, Wong TY, Tapp RJ, Harper CA, Zimmet PZ, Atkins R, Shaw JE, et al. Is peripheral neuropathy associated with retinopathy and albuminuria in individuals with impaired glucose metabolism? The 1999-2000 AusDiab. Diabetes Care. 2006;29(5):1114-1116.

8. Im S, Kim SR, Park JH, Kim YS, Park GY. Assessment of the medial dorsal cutaneous, dorsal sural, and medial plantar nerves in impaired glucose tolerance and diabetic patients with normal sural and superficial peroneal nerve responses. Diabetes Care. 2012;35(4):834-839.

9. Gordon Smith A, Robinson Singleton J. Idiopathic neuropathy, prediabetes and the metabolic syndrome. J Neurol Sci. 2006;242(1-2):9-14.

10. Viswanathan V, Seena R, Nair MB, Snehalatha C, Bhoopathy RM, Ramachandran A. Nerve conduction abnormalities in different stages of glucose intolerance. Neurol India. 2004;52(4):466-469.

11. Lupachyk S, Watcho P, Hasanova N, Julius U, Obrosova IG. Triglyceride, nonesterified fatty acids, and prediabetic neuropathy: role for oxidative-nitrosative stress. Free Radic Biol Med. 2012;52(8):1255-1263. 